\title{
Appendix to Compacting Boolean Formulae for Inference in Probabilistic Logic Programming
}

\author{
Theofrastos Mantadelis ${ }^{1}$, \\ Dimitar Shterionov ${ }^{2}$, Gerda Janssens ${ }^{2}$ \\ ${ }^{1}$ CRACS \& INESC TEC, \\ Faculty of Sciences, University of Porto \\ Rua do Campo Alegre 1021/1055, \\ 4169-007 Porto, Portugal \\ ${ }^{2}$ Department of Computer Science, \\ KU Leuven \\ Celestijnenlaan 200A, bus 2402, \\ 3001 Heverlee, Belgium \\ Email: theo.mantadelis@dcc.fc.up.pt Email: firstname.secondname@cs.kuleuven.be
}

\begin{abstract}
This appendix presents detailed description and analysis of our detection and compaction algorithm as well as the results from its empirical evaluation. We provide a logic-like pseudo-code to present our algorithm and give a thorough analysis of its complexity. Then we outline the benchmarks used for our experiments - we experimented on 7 benchmark sets with a total of 738 common ProbLog programs. We then present the results of our experiments and draw conclusions.
\end{abstract}

\section{A Introduction}

This document is a supplementary material to [6]. It presents a thorough analysis of our 6-pattern detection algorithm, description of the benchmark sets used for our experiments and detailed results.

In Table 1 we illustrate the 7 patterns we identify. We detect patterns 1 to 6 and use them to compact Boolean formulae.

\section{B Algorithm}

Our compaction algorithm is presented as a logic like pseudo-code in Algorithm 1. For our implementation we use AND/OR edges instead of AND/OR nodes. Boolean literals (Boolean variables/facts, introduced literals/subgoals) appear as nodes in our implementation. This algorithm detects patterns 1 to 6 ; the detection of pattern 7 is computationally expensive and for that reason we have chosen not to implement it. In one iteration of the algorithm we detect the patterns in the order that they are given in Table 1 . Once a pattern is detected we perform immediately the corresponding compaction before looking for the next pattern. This order allows more complex compactions to be detected earlier and compress the AND-OR graph in less iterations. We stop iterating when no pattern is detected. Note that in principle we could stop after any number of iterations. 


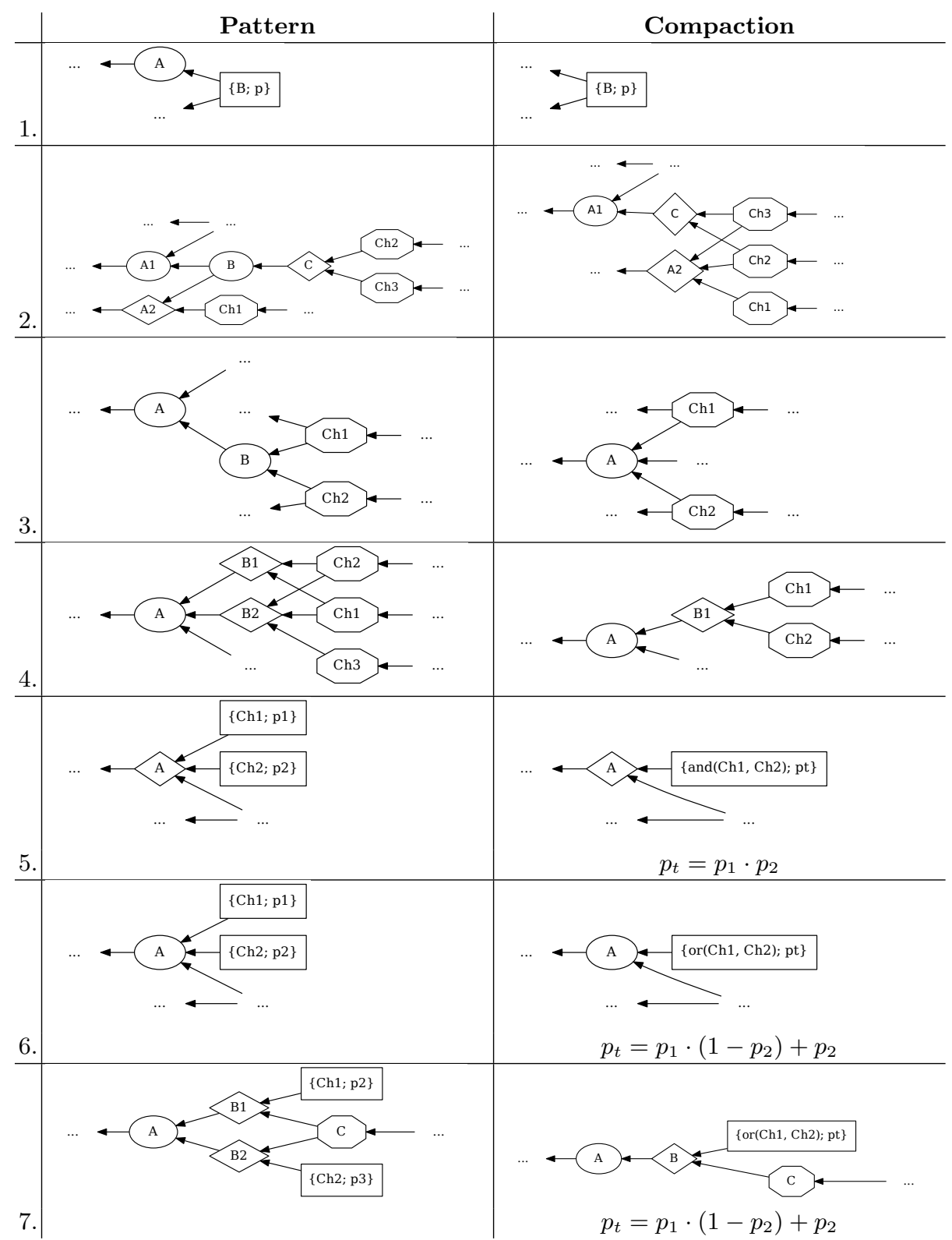

Table 1: AND-OR graph patterns and the compacting transformations. We denote with "..." multiple possible nodes to/from which exists an edge. With octagons we represent nodes that can be of any type (terminal, AND or OR). 
Data: An AND-OR graph

Result: Detected Node, Nodes to be compacted

detect_single_variable(NodeA, Terminal) $\leftarrow$

or_edge(NodeA, Terminal),

is_terminal(Terminal),

$\nexists$ and_edge(NodeA,_-_),

$\nexists$ (or_edge(NodeA, Any), Terminal $\neq$ Any).

detect_single_branch1(NodeB, NodeC) $\leftarrow$

or_edge(NodeB, NodeC),

and_edge(NodeC, _),

$\nexists$ and_edge(NodeB, _.),

$\nexists$ (or_edge(NodeB, Any), NodeC $\neq$ Any).

detect_single_branch2(NodeA, NodeB) $\leftarrow$

or_edge(NodeA, NodeB),

or_edge(NodeB, _),

$\nexists$ and_edge(_, NodeB),

$\nexists$ (or_edge(Any, NodeB), NodeA $\neq$ Any).

detect_minimal_proof(NodeB1, NodeB2) $\leftarrow$

or_edge(NodeA, NodeB1),

and_edge(NodeB1, _),

or_edge(NodeA, NodeB2),

and_edge(NodeB2, _),

NodeB1 $\neq$ NodeB2,

all(Child, and_edge(NodeB1, Child), ChildsB1),

all(Child, and_edge(NodeB2, Child), ChildsB2),

ChildsB $1 \subseteq$ ChildsB2.

detect_and_cluster(RefinedChilds) $\leftarrow$

and_edge(NodeA, _),

all(Terminal, (

and_edge(NodeA, Terminal),

is_terminal(Terminal),

$\nexists$ or_edge(_, Terminal)

), Childs),

get_all_and_edge_sets(ChildSets),

refine_cluster(ChildSets, Childs, RefinedChilds),

RefinedChilds $\neq \emptyset$.

detect_or_cluster1(RefinedChilds) $\leftarrow$

or_edge(NodeA, _),

all(Terminal, (

or_edge(NodeA, Terminal),

is_terminal(Terminal),

$\nexists$ and_edge(_, Terminal)

), Childs),

get_all_or_edge_sets(ChildSets),

refine_cluster(ChildSets, Childs, RefinedChilds),

RefinedChilds $\neq \emptyset$.

refine_cluster([], RefinedChilds, RefinedChilds).

refine_cluster([Set|ChildSets], Childs, RefinedChilds) $\leftarrow$

NewChilds $=$ Set $\wedge$ Childs,

refine_cluster(ChildSets, NewChilds, RefinedChilds).

Algorithm 1: The 6 pattern detection algorithms. 


\section{B.1 Analysis}

Complexity: Our implementation uses indexed tables to represent the ANDOR graph. Finding an edge in the graph takes logarithmic time, inserting an edge or deleting an edge (after finding it) is performed in constant time. That is why, compacting a detected pattern is very efficient $(O(N)$ with $N$ the number of edges affected). Detecting a pattern is the bottleneck of our algorithm and deserves further analysis.

For an arbitrary AND-OR graph $G$ we denote with $N_{\text {or }}$ the number of $O R$ edges, with $N_{\text {and }}$ the number of $A N D$ edges and with $N_{\text {term }}$ the number of terminal nodes. We assume that a node always contains $N_{\text {term }}$ children; this is a high upper bound assumption but does not affect the complexity class.

Patterns 1 to 3 have similar worst case complexity. Notice that Alg. 1 performs similar operations in order to verify that an OR edge participates in one of the patterns. For detecting all patterns 1 in an AND-OR graph we are required to check each OR edge from a terminal node $B$ to a node $A$. We verify that node $A$ is forming a pattern 1 with node $B$ by checking that no other OR/AND edge exists to a second node different than the terminal node $B$. Complexity: $O\left(\log \left(N_{\text {or }}\right)+\log \left(N_{\text {and }}\right)\right)$ for verifing one pattern; $O\left(N_{\text {or }} \cdot\left(\log \left(N_{\text {or }}\right)+\log \left(N_{\text {and }}\right)\right)\right)$ to detect all patterns. Similarly, for patterns 2 and 3.

Pattern 4 checks for each OR edge in the AND/OR graph whether it is connected with two different literals that have AND edges and form two sets that one is a subset of the other. The collection of the children and set comparisons are made in linear time to the number of children. Complexity: $O\left(N_{o r} \cdot\left(\log \left(N_{o r}\right)+\right.\right.$ $\left.\left.\log \left(N_{\text {and }}\right)+N_{\text {term }}\right)\right)$.

For detecting all AND-Clusters we need to collect for each AND edge all its children that are terminal nodes and not contained in OR edges, thus creating $N_{\text {and }}$ sets of children. Finally, we need for each of these sets to refine it with all the other sets in order to create a common subset. All the set operations are linear to the size of the largest set. Collecting all the nodes takes $O\left(N_{\text {and }} \cdot N_{\text {term }}\right)$. Refining a candidate AND-Cluster takes $O\left(N_{\text {and }} \cdot N_{\text {term }}\right)$. The total complexity is: $O\left(N_{\text {and }}^{2} \cdot N_{\text {term }}\right)$.

OR-Cluster I. The analysis of the complexity of detecting an OR-Cluster I is similar to the one for AND-Clusters. Detecting OR-Clusters considers OR edges. Therefore, the complexity bound is $O\left(N_{o r}^{2} \cdot N_{t e r m}\right)$.

OR-Cluster II. This pattern is not implemented in our code. Here we give a theoretical complexity for the simple case (size 2) depicted in Table 1. We need to check all OR edges and see that they have two children that participate as parents to two different AND edges. Then with a set operator we can find the common part and need to check that exactly one child remains. Finally, we need to search all the graph for the child not to appear anywhere else. Total complexity for size 2: $O\left(N_{\text {or }} \cdot N_{\text {term }}\left(N_{\text {or }}+N_{\text {and }}\right)\right)$ we believe the complexity increases polynomially for bigger sizes. 


\section{Benchmarks}

To experiment with our compaction algorithm we use 738 ProbLog programs from 7 different benchmark sets. We perform experiments with the two basic probabilistic inference tasks: (i) the MARG task, i.e. the task to compute the probability a query is true for a give ProbLog program; and (ii) the COND task, i.e. the task to compute the conditional probability of a query given evidence. The benchmark sets are summarized in Table 2 a detailed description follows.

\begin{tabular}{|c|c|c|c|c|c|c|c|}
\hline & Name: & Generated from: & $\begin{array}{c}\text { Number of } \\
\text { benchmark } \\
\text { sets: }\end{array}$ & $\begin{array}{c}\text { Number of } \\
\text { programs } \\
\text { in one set: }\end{array}$ & $\mid \begin{array}{c}\text { Total } \\
\text { number of } \\
\text { programs: }\end{array}$ & Cyclic: & $\begin{array}{c}\text { Inference } \\
\text { task: }\end{array}$ \\
\hline 1. & Alzheimer & Real-world data & 6 & 17 & 102 & Yes & MARG \\
\hline 2 . & Balls & Artificial data & 1 & 150 & 150 & No & MARG \\
\hline 3. & Dictionary & Real-world data & 1 & 100 & 100 & Yes & MARG \\
\hline 4. & Grid & Artificial data & 1 & 14 & 14 & No & MARG \\
\hline 5 . & Les Miserables & Real-world data & 1 & 60 & 60 & Yes & MARG \\
\hline 6. & Smokers & Artificial data & 5 & 24 & 120 & Yes & COND \\
\hline 7. & WebKB & Real-world data & 4 & 48 & 192 & Yes & COND \\
\hline
\end{tabular}

Table 2: Summary of the benchmarks used in our experiments.

1. The Alzheimer benchmark set [1] is build from a real-world biological dataset of Alzheimer genes. The data is represented as a directed probabilistic graph with 11530 edges and 5220 nodes. We used 17 subgraphs with increasing sizes and without duplicate edges extracted from the initial graph. We used 6 different path queries for each of the (sub)graphs. With each combination query-graph we associate one ProbLog program.

2. The Balls benchmark set, presented in [10, contains ProbLog programs encoding a game in which a player draws colorful balls (red, green and blue) from different bags one bag after another. The player can choose only one ball per bag. To be able to select from a bag the previous selections should fulfill certain conditions. The different options for a bag are encoded as annotated disjunctions 11/7. We use 150 different queries that compute the probability a ball is selected from a specific bag. Each query is associated with a separate ProbLog program.

3. The probabilistic Dictionary benchmark set (9) includes around 250 different words from the English language. They are linked to each according to a similarity measure expressed with a probability (probability 1.0 states that two words mean exactly the same; probability 0.0 that two words do not mean the same). The probabilities are computed according to two approaches: (i) the algorithm presented in [9] and (ii) MSR (http://cwl-projects.cogsci. rpi.edu/msr/). They form an incomplete probabilistic graph. For 30 of the words their meaning is also given. We use 100 randomly selected queries which look for the probability that two words have the same meaning even if an explicit link has not been defined. There are more than 7000 possible combinations. Each query is associated with one ProbLog program. 
4. 3. introduces the probabilistic Grid as a special case of a probabilistic graph. We use a grid with $25 \times 25$ nodes. Each node $n_{x, y}$ is connected by a directed edge to the nodes $n_{x+1, y}, n_{x, y+1}$ and $n_{x+1, y+1}$. We use different queries $\operatorname{path}\left(1, n_{x, x}\right)$ where $x=2, . ., 15$.

5. The Les Miserables [5] originally is a deterministic dataset presenting the relations of the characters from the same-name novel who appeared in the same chapter. The data was shifted to a probabilistic setting by calculating the probability that two randomly selected characters will appear in the same chapter i.e. a tie relation. We use 60 benchmark programs each containing one query. A single query asks for the probability of a tie between two characters.

6. Smokers 8 is a dataset which expresses a friend network. Each person can smoke either because of stress or because he/she is influenced by a friend who smokes. In a ProbLog program from the "Smokers" benchmark set, the influence relations are encoded as probabilistic facts. This ProbLog program contains a large number of loops. We use programs with an increasing number of people: from 3 until 27 which is indicative for the size of the program. One benchmark program contains multiple queries and evidence. A single query asks the probability that a person smokes given that the evidence holds. We use this set to compute conditional probabilities. We use 5 random instances of this benchmark set.

7. The WebKB benchmark set is built upon a dataset from a collective classification domain in which university webpages are classified according to their textual content (http://www.cs.cmu.edu/ webkb/) 2]. All the probabilities are learned from data 4. We 4 instances with 48 programs containing different sets of queries and evidence atoms. We compute the conditional probability of queries being true given that the evidence holds.

Our benchmarks have been used previously to evaluate different aspects of ProbLog implementations. The benchmarks from the "Alzheimer" set were used to motivate the development and test the performance of the first ProbLog system. The "Smokers" and "WebKB" benchmark sets are used for testing ProbLog2, i.e. different loop-breaking and knowledge compilation approaches. Also, the "Grid" benchmark set was developed in the context of ProbLog2 and to compare the knowledge compilation to sd-DNNFs with knowledge compilation to ROBDDs. The "Balls" benchmark set is used to test the performance of a new encoding of Annotated Disjunctions for ProbLog programs (mainly affecting the grounding). That is why we believe our experiments allow to clearly determine the actual effect of our compaction algorithm on probabilistic inference.

\section{Experimental Results}

In our experiments we collect the total run time for executing a benchmark program (including the compaction time). We use the time results to determine the compaction setting which leads to (a) the lowest run times; and (b) to the lowest number of time-outs for each of the pipelines and each benchmark set. 
Effect on knowledge compilation: We present the number of benchmark programs for which knowledge compilation performs better when compaction $(\{$ prior, post, both $\})$ is enabled then otherwise in Fig. 1. For each ProbLog pipeline and each compaction setting $c \in\{$ prior, post, both $\}$ we count (a) the number of programs for which knowledge compilation performs better compared to no compaction setting $(c=$ none); (b) the number of programs for which the performance of the knowledge compilation is the same - within a $5 \%$ insignificance interval; and (c) the number of programs for which knowledge compilation when $c=$ none performs better. We exclude programs for which both the inference with compaction and with no compaction times out. We give these numbers as percentages. For clarity we show only (a) and (b); (c) is the remaining up to $100 \%$. Compaction improves the majority of the executed benchmarks. Its effect

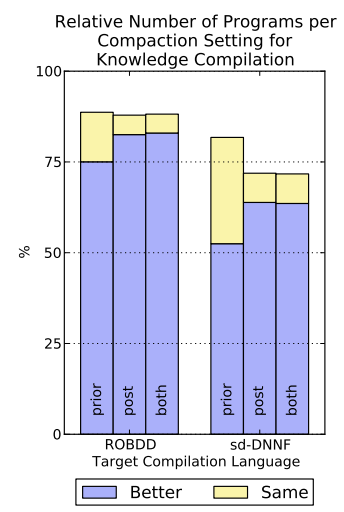

Fig. 1: Relative number of programs for which knowledge compilation performs best with the different compaction settings.

is more salient for knowledge compilation to ROBDDs than to sd-DNNs. One reason is the heuristics employed by the compiler. It is often the case that redundant information can improve a heuristic search but our algorithm removes any such information aiming at the most compact formula.

Best performing compaction setting: For each ProbLog pipeline and each compaction setting $c \in\{$ prior, post, both $\}$ we count (a) the number of programs that perform better compared to no compaction setting $(c=$ none); (b) the number of programs for which the performance is the same - within a $5 \%$ insignificance interval; and (c) the number of programs for which $c=$ none performs better. We exclude programs for which both the inference with compaction and with no compaction times out. We give these numbers as percentages in Fig. 2 For clarity we show only (a) and (b); (c) is the remaining up to $100 \%$.

Fig. 2 does not quantify the gain or loss due to compaction just presents percentile wise how many programs gain from compaction. In order to estimate the overall gain due to compaction in Fig. 3 we plot the following measurement: for each ProbLog pipeline and each compaction $c \in\{$ prior, post, both $\}$ we sum (a) the gain in the total run time for each benchmark compared with the run time of no compaction ( $c=$ none) when the compaction performs better: $T_{C}^{g}$ (this is the 

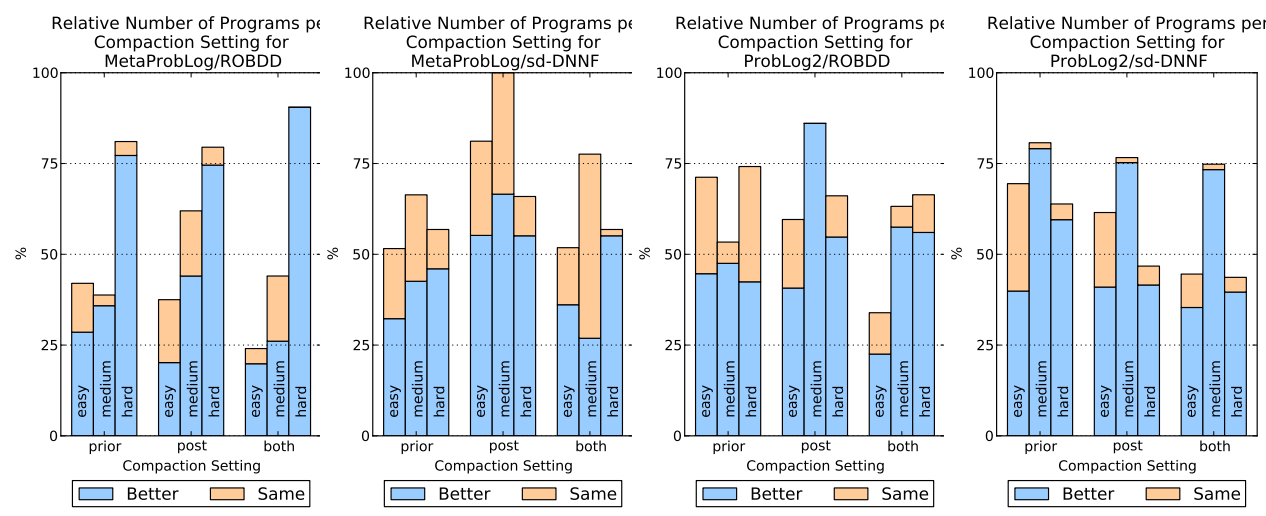

Fig. 2: Relative number of programs with best total run time for the different compaction settings.
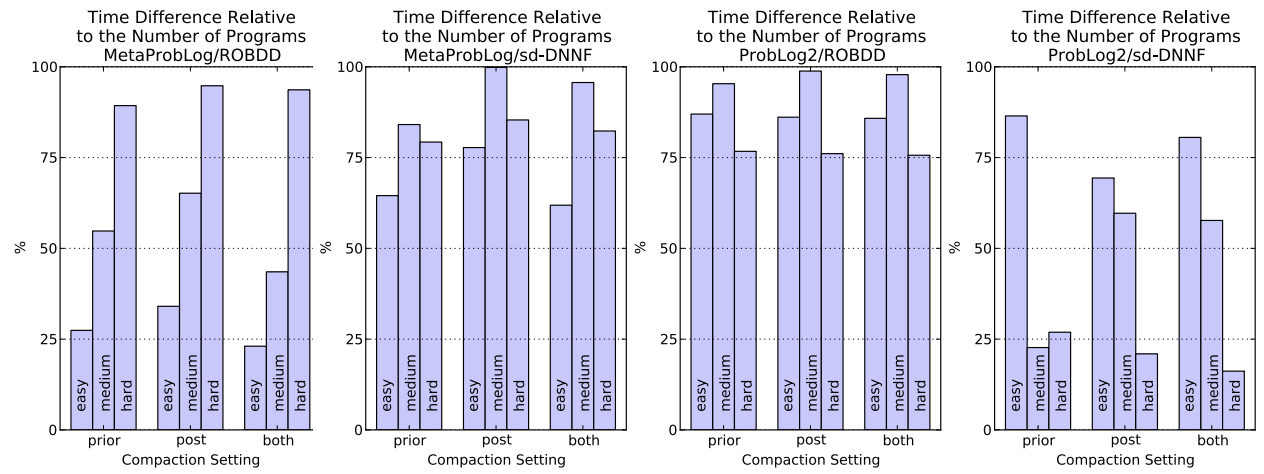

Fig. 3: Relative time gain due to a specific compaction.

total gain for compaction); (b) the gain in the total run time when no compaction performs better: $T_{N}^{g}$ (this is the total loss for compaction). We normalize each gain by dividing by the total number of programs within a benchmark set to compensate for the fact that some of them contain more programs. For example, consider a particular benchmark with programs $\left\{b_{1}, b_{2}, b_{3}\right\}$ each with run times: with compaction 2030 and 40 seconds and with no compaction 10, 25, 70 seconds. Then $T_{C}^{g}=70-40=30$ and $T_{N}^{g}=(20-10)+(30-25)=15$ showing that in total the gain with compaction exceeds the loss due to compaction. We exclude programs for which both inference with compaction and with no compaction times out. We show the gain due to compaction relative to the total gain: $\frac{T_{C}^{g}}{T_{C}^{g}+T_{N}^{g}}$ in Fig. 3 These results illustrate the run time gain due to compaction in percentage. 
Least time-outs compaction setting: For each benchmark set $d$ we count the total number of programs solved within the 540 seconds by at least one pipeline and compaction setting (we denote this number as $S_{d}$ ); for each pipeline $p$ and compaction setting $c$ we count the number of programs from the benchmark set $d$ which time out $-T_{d, p, c}$. We ignore the programs for which all pipelines time-out. We also compute the total number of programs which time out for one pipeline and one compaction setting: $\sum_{d \in D} T_{d, p, c}$; and we compute the accumulated ratio $\sum_{d \in D} \frac{T_{d, p, c}}{S_{d}}$, where $D$ is the set of all benchmark sets. For the "Les Miserables", "Smokers 1", "Smokers 2" and "Smokers 4" sets there is no one pipeline that succeeds over all 636 programs we managed to solve. The lowest number of time-outs for these cases are larger than 0 . For these programs at least one of the other pipelines does succeed within the 540 seconds. These results are shown in Table 3 .

\begin{tabular}{|c|c|c|c|c|c|c|c|c|c|c|c|c|c|c|c|c|c|}
\hline Benchmark & 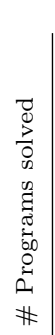 & 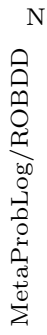 & 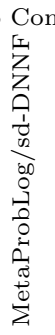 & 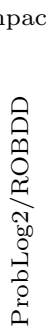 & 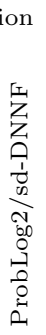 & 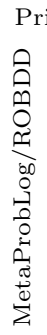 & 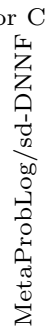 & 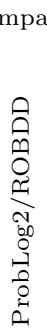 & 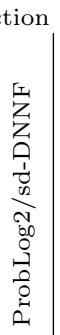 & 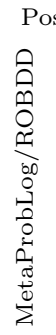 & 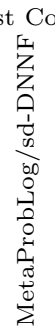 & 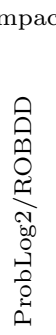 & 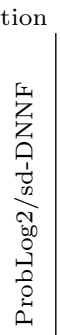 & 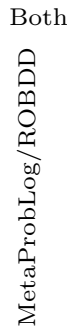 & 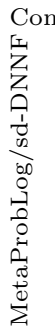 & 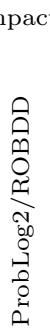 & 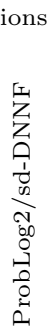 \\
\hline alzheimer_q1 & 5 & 2 & 4 & 1 & 4 & 2 & 4 & 0 & 4 & 1 & 4 & 1 & 4 & 1 & 4 & 1 & 4 \\
\hline alzheimer_q2 & 9 & 4 & 6 & $\mathbf{0}$ & 5 & 3 & 6 & $\mathbf{0}$ & 5 & 3 & 6 & $\mathbf{0}$ & 5 & 3 & 6 & $\mathbf{0}$ & 6 \\
\hline alzheimer_q3 & 9 & 0 & 0 & 3 & 3 & 0 & 0 & 3 & 3 & 0 & $\mathbf{0}$ & 3 & 3 & $\mathbf{0}$ & 0 & 3 & 3 \\
\hline alzheimer_q4 & 17 & 0 & $\mathbf{0}$ & 0 & 0 & 0 & 0 & 0 & $\mathbf{0}$ & 0 & $\mathbf{0}$ & $\mathbf{0}$ & 0 & $\mathbf{0}$ & 0 & 0 & 0 \\
\hline alzheimer_q5 & 9 & 3 & 3 & 1 & 5 & 3 & 3 & 3 & 5 & 1 & 3 & 3 & 4 & $\mathbf{0}$ & 3 & 3 & 4 \\
\hline alzheimer_q6 & 6 & 1 & 3 & 0 & 2 & 0 & 3 & 0 & 2 & 0 & 3 & $\mathbf{0}$ & 2 & $\mathbf{0}$ & 3 & 0 & 2 \\
\hline balls & 150 & 0 & $\mathbf{0}$ & 35 & 0 & 0 & 0 & 13 & $\mathbf{0}$ & $\overline{0}$ & 0 & $\mathbf{0}$ & 0 & 0 & $\mathbf{0}$ & $\overline{0}$ & 0 \\
\hline dictionary & 100 & 0 & 0 & 0 & 0 & 0 & $\mathbf{0}$ & 0 & 0 & 0 & 0 & $\mathbf{0}$ & 0 & $\mathbf{0}$ & 0 & $\mathbf{0}$ & 0 \\
\hline grid & 12 & 4 & 0 & 1 & 0 & 4 & 2 & 1 & 1 & 4 & 1 & 1 & 1 & 4 & 1 & 1 & 1 \\
\hline les_miserables & 47 & 2 & 10 & 2 & 27 & 2 & 14 & 2 & 24 & 2 & 16 & 3 & 26 & 2 & 21 & 2 & 28 \\
\hline smokers1 & 20 & 6 & 13 & 0 & 3 & 6 & 12 & $\mathbf{0}$ & 3 & 7 & 13 & $\mathbf{0}$ & 3 & 6 & 12 & $\mathbf{0}$ & 3 \\
\hline smokers2 & 20 & 5 & 16 & 1 & 3 & 5 & 16 & $\mathbf{0}$ & 3 & 5 & 16 & 1 & 3 & 5 & 16 & 1 & 3 \\
\hline smokers3 & 18 & 3 & 11 & 0 & 1 & 3 & 13 & $\mathbf{0}$ & 1 & 4 & 12 & $\mathbf{0}$ & 1 & 3 & 13 & $\mathbf{0}$ & 1 \\
\hline smokers4 & 20 & 5 & 13 & 1 & 3 & 3 & 14 & 1 & 3 & 5 & 14 & 1 & 4 & 3 & 15 & 1 & 4 \\
\hline smokers5 & 17 & 1 & 12 & 0 & 0 & 2 & 12 & $\mathbf{0}$ & 0 & 1 & 12 & 0 & 0 & 3 & 11 & 0 & 0 \\
\hline webkb1 & 48 & 18 & 29 & 9 & 0 & 10 & 14 & 3 & 0 & 8 & 14 & 3 & 0 & 8 & 14 & 4 & 3 \\
\hline webkb2 & 33 & 8 & 19 & 8 & 3 & 8 & 13 & 7 & 0 & 8 & 13 & 8 & 2 & 8 & 14 & 8 & 2 \\
\hline webkb4 & 48 & 20 & 41 & 17 & 0 & 7 & 41 & 1 & 12 & 1 & 41 & 1 & 14 & 0 & 41 & 1 & 10 \\
\hline webkb5 & 48 & 17 & 29 & 10 & 0 & 8 & 14 & 4 & 0 & 7 & 14 & 7 & 0 & 9 & 14 & 7 & 3 \\
\hline Total: & 636 & 99 & 209 & 89 & $\underline{59}$ & 66 & 181 & 38 & 66 & 57 & 182 & 32 & 72 & 55 & 188 & $\underline{32}$ & 77 \\
\hline
\end{tabular}
Table 3: Number of time-outs. In bold font we mark the lowest number of timeouts per benchmark set; with underlined we mark the lowest sum and lowest accumulated ratio of the time-outs for a pipeline and a compaction setting.

Compaction Time Our algorithm has a polynomial complexity (see Section B.1. Here we show the actual time that is consumed by our detection and 
compaction algorithm. In Table 4 and Table 5 we present the minimum and maximum times consumed by the prior, post or both compactions versus the total run time per benchmark for grounding to Ground LP and to Nested Tries accordingly. That is, for each benchmark set we collect the time for compaction $T_{C}$ (for prior, post and both) as well as the total time $T_{t o t}$. Then we determine the programs which have the lowest and the highest compaction time $T_{C}^{\min }$ and $T_{C}^{\max }$ and their total run times $T_{\text {tot }}^{\min }$ and $T_{\text {tot }}^{\max }$. Then we show the tuples $T_{C}^{\min } / T_{\text {tot }}^{\min }$ and $T_{C}^{\max } / T_{\text {tot }}^{\max }$ in the tables. Table 4 and Table 5 show that the

\begin{tabular}{c|c|c|c|c|c|c} 
& \multicolumn{2}{|c|}{ Prior } & \multicolumn{2}{c|}{ Post } & \multicolumn{2}{c}{ Both } \\
\hline \hline Benchmark & Min & Max & Min & Max & Min & Max \\
\hline alzheimer_q1 & $1.32 / 2.08$ & $3.94 / 519.63$ & $0.19 / 0.88$ & $9.42 / 23.69$ & $1.5 / 2.02$ & $11.87 / 24.19$ \\
alzheimer_q2 & $1.42 / 1.72$ & $7.95 / 451.17$ & $0.09 / 0.39$ & $10.29 / 257.59$ & $1.52 / 1.74$ & $18.25 / 264.27$ \\
alzheimer_q3 & $0.1 / 0.17$ & $3.93 / 268.92$ & $0.05 / 0.16$ & $0.26 / 382.5$ & $0.14 / 0.21$ & $3.98 / 269.76$ \\
alzheimer_q4 & $0.05 / 0.1$ & $0.24 / 0.46$ & $0.04 / 0.13$ & $0.04 / 0.3$ & $0.09 / 0.14$ & $0.28 / 0.48$ \\
alzheimer_q5 & $0.11 / 0.24$ & $0.5 / 267.79$ & $0.05 / 0.2$ & $1.87 / 113.28$ & $0.16 / 0.26$ & $2.37 / 113.61$ \\
alzheimer_q6 & $1.4 / 1.68$ & $3.32 / 92.3$ & $0.1 / 0.4$ & $5.78 / 131.44$ & $1.48 / 1.71$ & $10.79 / 136.35$ \\
balls & $0.04 / 0.08$ & $3.83 / 93.48$ & $0.04 / 0.12$ & $6.96 / 76.37$ & $0.09 / 0.13$ & $7.48 / 77.24$ \\
dictionary & $0.04 / 0.08$ & $0.05 / 1.4$ & $0.04 / 0.13$ & $0.04 / 1.09$ & $0.09 / 0.13$ & $0.09 / 1.1$ \\
grid & $0.06 / 0.15$ & $0.11 / 107.21$ & $0.05 / 0.21$ & $0.15 / 100.54$ & $0.11 / 0.22$ & $0.21 / 438.91$ \\
les_miserables & $0.04 / 0.08$ & $0.08 / 534.84$ & $0.04 / 0.12$ & $0.42 / 222.54$ & $0.08 / 0.13$ & $1.57 / 369.71$ \\
smokers1 & $0.03 / 0.07$ & $0.05 / 173.61$ & $0.03 / 0.1$ & $0.09 / 133.69$ & $0.06 / 0.1$ & $0.13 / 202.9$ \\
smokers2 & $0.04 / 0.08$ & $0.06 / 538.9$ & $0.04 / 0.13$ & $0.25 / 531.27$ & $0.08 / 0.13$ & $0.28 / 531.63$ \\
smokers3 & $0.04 / 0.1$ & $0.06 / 190.54$ & $0.04 / 0.15$ & $0.58 / 301.3$ & $0.09 / 0.15$ & $0.59 / 299.9$ \\
smokers4 & $0.04 / 0.08$ & $0.05 / 360.08$ & $0.04 / 0.13$ & $0.6 / 464.78$ & $0.08 / 0.13$ & $0.58 / 462.04$ \\
smokers5 & $0.04 / 0.1$ & $0.06 / 74.4$ & $0.04 / 0.14$ & $0.08 / 26.18$ & $0.09 / 0.14$ & $0.14 / 21.59$ \\
webkb1 & $0.05 / 0.79$ & $1.11 / 340.08$ & $0.05 / 0.86$ & $1.25 / 414.36$ & $0.09 / 0.84$ & $1.45 / 359.86$ \\
webkb2 & $0.04 / 0.73$ & $0.29 / 237.8$ & $0.04 / 0.78$ & $2.04 / 92.82$ & $0.08 / 0.77$ & $0.85 / 103.56$ \\
webkb4 & $0.05 / 0.74$ & $0.64 / 242.11$ & $0.04 / 0.81$ & $0.39 / 377.47$ & $0.09 / 0.79$ & $1.28 / 486.33$ \\
webkb5 & $0.05 / 0.79$ & $0.72 / 434.88$ & $0.05 / 0.85$ & $1.28 / 295.33$ & $0.1 / 0.85$ & $1.43 / 317.09$
\end{tabular}

Table 4: Minimum and maximum compaction time versus total run time for AND-OR graphs generated from Ground LPs.

time for compaction is in practice small. Its maximum of 64.95 seconds in the case of Nested Ties, both compaction setting and for "Smokers2" benchmark set (see Table 5) is in practice around $20 \%$ of the total runtime. Furthermore, we notice that the compaction cost for inference pipelines that use a Ground LP is generally smaller than for inference pipelines using nested tries. 


\begin{tabular}{c|c|c|c|c|c|c} 
& \multicolumn{2}{|c|}{ Prior } & \multicolumn{2}{c|}{ Post } & \multicolumn{2}{c}{ Both } \\
\hline \hline Benchmark & Min & Max & Min & Max & Min & Max \\
\hline alzheimer_q1 & $1.04 / 2.66$ & $2.0 / 17.51$ & $0.07 / 0.58$ & $17.69 / 139.28$ & $1.11 / 2.68$ & $20.38 / 141.88$ \\
alzheimer_q2 & $1.13 / 2.54$ & $4.17 / 331.08$ & $0.02 / 0.14$ & $7.82 / 308.4$ & $1.15 / 2.54$ & $11.3 / 259.23$ \\
alzheimer_q3 & $0.05 / 0.13$ & $5.51 / 321.67$ & $0.0 / 0.03$ & $0.0 / 281.88$ & $0.05 / 0.13$ & $5.54 / 302.82$ \\
alzheimer_q4 & $0.0 / 0.03$ & $0.16 / 0.54$ & $0.0 / 0.02$ & $0.0 / 0.18$ & $0.0 / 0.03$ & $0.16 / 0.52$ \\
alzheimer_q5 & $0.05 / 0.14$ & $0.38 / 158.32$ & $0.01 / 0.04$ & $0.24 / 230.54$ & $0.05 / 0.14$ & $3.15 / 438.45$ \\
alzheimer_q6 & $1.13 / 2.53$ & $4.15 / 327.9$ & $0.02 / 0.14$ & $7.79 / 345.25$ & $1.17 / 2.57$ & $11.21 / 240.1$ \\
balls & $0.0 / 0.02$ & $5.99 / 78.89$ & $0.0 / 0.02$ & $6.07 / 78.4$ & $0.0 / 0.02$ & $8.77 / 84.84$ \\
dictionary & $0.0 / 0.02$ & $0.01 / 1.22$ & $0.0 / 0.02$ & $0.01 / 1.87$ & $0.0 / 0.02$ & $0.02 / 1.66$ \\
grid & $0.0 / 0.02$ & $0.02 / 271.24$ & $0.0 / 0.02$ & $0.03 / 110.92$ & $0.0 / 0.02$ & $0.09 / 149.19$ \\
les_miserables & $0.0 / 0.02$ & $0.0 / 178.72$ & $0.0 / 0.02$ & $0.26 / 95.19$ & $0.0 / 0.02$ & $0.26 / 94.95$ \\
smokers1 & $0.0 / 0.02$ & $0.01 / 436.57$ & $0.0 / 0.02$ & $3.68 / 108.58$ & $0.0 / 0.02$ & $38.45 / 404.6$ \\
smokers2 & $0.0 / 0.02$ & $0.01 / 147.54$ & $0.0 / 0.02$ & $29.98 / 201.61$ & $0.0 / 0.02$ & $64.95 / 297.79$ \\
smokers3 & $0.0 / 0.02$ & $0.01 / 64.96$ & $0.0 / 0.03$ & $8.02 / 98.45$ & $0.0 / 0.03$ & $25.36 / 117.75$ \\
smokers4 & $0.0 / 0.03$ & $0.01 / 368.1$ & $0.0 / 0.03$ & $20.93 / 306.63$ & $0.0 / 0.03$ & $21.8 / 352.89$ \\
smokers5 & $0.0 / 0.02$ & $0.01 / 311.78$ & $0.0 / 0.02$ & $7.87 / 354.27$ & $0.0 / 0.02$ & $0.02 / 140.88$ \\
webkb1 & $0.01 / 0.06$ & $1.94 / 370.12$ & $0.01 / 0.05$ & $3.93 / 259.48$ & $0.01 / 0.05$ & $4.72 / 479.17$ \\
webkb2 & $0.0 / 0.03$ & $0.17 / 411.62$ & $0.0 / 0.03$ & $1.35 / 453.89$ & $0.0 / 0.03$ & $0.64 / 423.95$ \\
webkb4 & $0.0 / 0.03$ & $2.77 / 466.59$ & $0.0 / 0.03$ & $8.46 / 101.19$ & $0.0 / 0.03$ & $15.47 / 174.75$ \\
webkb5 & $0.01 / 0.08$ & $1.97 / 413.38$ & $0.01 / 0.06$ & $9.66 / 443.91$ & $0.01 / 0.07$ & $5.34 / 195.45$
\end{tabular}

Table 5: Minimum and maximum compaction time versus total run time for AND-OR graphs generated from Nested Tries.

\section{References}

1. L. De Raedt, A. Kimmig, and H. Toivonen. ProbLog: A probabilistic Prolog and its application in link discovery. In IJCAI, pages 2468-2473. AAAI Press, 2007.

2. D. Fierens, G. Van den Broeck, I. Thon, B. Gutmann, and L. De Raedt. Inference in probabilistic logic programs using weighted CNFs. In UAI, pages 211-220, 2011.

3. Daan Fierens, Guy Van den Broeck, Joris Renkens, Dimitar Sht. Shterionov, Bernd Gutmann, Ingo Thon, Gerda Janssens, and Luc De Raedt. Inference and learning in probabilistic logic programs using weighted boolean formulas. TPLP, 15(3):358401, 2015.

4. Bernd Gutmann. On Continuous Distributions and Parameter Estimation in Probabilistic Logic Programs. PhD thesis, Informatics Section, Department of Computer Science, Faculty of Engineering Science, October 2011. De Raedt, Luc (supervisor).

5. D. Knuth. The Stanford GraphBase: A Platform for Combinatorial Computing. Addison-Wesley, MA, 1993.

6. Theofrastos Mantadelis, Dimitar Shterionov, and Gerda Janssens. Compacting boolean formulae for inference in probabilistic logic programming. In Proceedings of the 13th International Conference on Logic Programming and Nonmonotonic Reasoning (LPNMR 2015), page 13, 27-30 September 2015. To appear.

7. Wannes Meert, Jan Struyf, and Hendrik Blockeel. CP-logic theory inference with contextual variable elimination and comparison to BDD based inference methods. In Proc. 19th International Conf. of Inductive Logic Programming, 2009. 
8. Luc De Raedt, Paolo Frasconi, Kristian Kersting, and Stephen Muggleton, editors. Probabilistic Inductive Logic Programming - Theory and Applications, volume 4911 of Lecture Notes in Computer Science. Springer, 2008.

9. Dimitar Shterionov and Gerda Janssens. Data acquisition and modeling for learning and reasoning in probabilistic logic environment. In Luis Antunes, H. Sofia Pinto, Rui Prada, and Paulo Trigo, editors, Proceedings of the 15th Portuguese Conference on Artificial Intelligence, pages 298-312, 2011.

10. Dimitar Shterionov and Gerda Janssens. Crucial components in probabilistic inference pipelines: Data and results. Technical report, KU Leuven, 2014. Ref. number CW679. Available at http://www.cs.kuleuven.be/publicaties/rapporten/cw/ CW679.pdf

11. Joost Vennekens, Sofie Verbaeten, and Maurice Bruynooghe. Logic programs with annotated disjunctions. In Proceedings of International Conference on Logic Programming, pages 431-445. Springer, 2004. 